\title{
An Algebraic Approach to Guarantee Harmonic Balance Method Using Gröbner Base
}

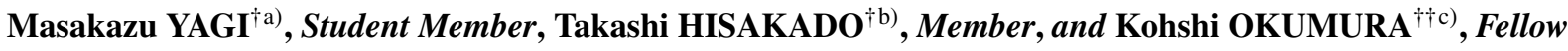

SUMMARY Harmonic balance (HB) method is well known principle for analyzing periodic oscillations on nonlinear networks and systems. Because the HB method has a truncation error, approximated solutions have been guaranteed by error bounds. However, its numerical computation is very time-consuming compared with solving the HB equation. This paper proposes an algebraic representation of the error bound using Gröbner base. The algebraic representation enables to decrease the computational cost of the error bound considerably. Moreover, using singular points of the algebraic representation, we can obtain accurate break points of the error bound by collisions.

key words: harmonic balance method, error bound, Gröbner base, algebraic representation, quadratic approximation, singular point

\section{Introduction}

Harmonic balance (HB) method is well known principle for analyzing periodic oscillations of nonlinear networks and systems [1], [2]. Using this method, we express circuit equations as simultaneous algebraic equations called HB equation due to an approximation by truncated Fourier series of variables. Although the HB method ignores high frequency components, the HB method enables to clarify essential relations among the system parameters. Recently, some techniques by the HB method for bifurcation analysis have been proposed [3]-[6].

Because the HB method has a truncation error, approximated solutions of the HB equation have been guaranteed by bounded regions, called error bounds, within which the solution must reside [7]-[9]. In particular, Swern presented a method to obtain the error bound for a feedback system with a polynomial-type nonlinear element [9]. However, the numerical computation of the error bound is very timeconsuming because we have to express the high dimensional error bound using a set of the numerical values.

In order to overcome the difficulty, we propose an algebraic representation of the error bound using Gröbner base [10], [11]. Several reports have proposed methods to apply Gröbner base to nonlinear circuit systems [12]-[15]. Gröbner base enables to eliminate variables from polyno-

Manuscript received November 27, 2007.

Manuscript revised March 12, 2008.

${ }^{\dagger}$ The authors are with the Department of Electrical Engineering, Kyoto University, Kyoto-shi, 615-8510 Japan.

${ }^{\dagger \dagger}$ The author is with the Department of Computer Science, Hiroshima Institute of Technology, Hiroshima-shi, 731-5193 Japan.

a) E-mail: yagi@ circuit.kuee.kyoto-u.ac.jp

b) E-mail: hisakado@kuee.kyoto-u.ac.jp

c)E-mail: kohshi@iis.it-hiroshima.ac.jp

DOI: $10.1093 /$ ietfec/e91-a.9.2442 mial simultaneous equations containing parameters [16], [17]. By Gröbner base, the algebraic representation of the error bound is described as only one algebraic equation with system parameters. The proposed method does not depend on the number of specific frequency components. Further, linear elements of the system are contained as parameters. Thus, when we fix the nonlinear elements, the algebraic representation can be uniquely obtained.

In order to visualize the error bound, we project the error bound to a complex plane of a target frequency component using the algebraic representation. However, the computation of its projection is time-consuming. Thus, we propose an approximated error bound using the algebraic representation. Although the proposed error bound approximately guarantees solutions, the approximated error bound reduces the computational cost of the projection considerably [11].

When we set the system parameters close to bifurcation parameters, there exist two error bounds in a neighborhood. In such cases, the error bounds are broken by a collision of each other. Hence, we cannot guarantee the solutions near the bifurcation point. The collision point is a break point which generates a singular point of the error bound. Since the singular point can be calculated by the algebraic representation, we propose a method to obtain the accurate break point of the error bound.

\section{Harmonic Balance (HB) Method and Error Bound}

\section{$2.1 \quad$ HB Method}

We consider the nonlinear feedback system shown in Fig. 1. The system equation is described by

$$
\begin{aligned}
& u(\tau)=G_{1}(s ; \boldsymbol{\mu})\left\{v(\tau)-G_{2}(s ; \boldsymbol{\mu}) N[u(\tau)]\right\}, \\
& N[u]=\sum_{i=0}^{p} c_{2 i+1} u^{2 i+1}, \quad c_{2 i+1}>0, \quad i=0, \ldots, p,
\end{aligned}
$$

where $s=\mathrm{d} / \mathrm{d} \tau, v(\tau)$ is an input function with a period $2 \pi$,

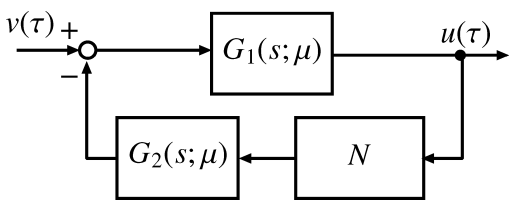

Fig. 1 Nonlinear feedback system. 
$\boldsymbol{\mu}=\left(\mu_{1}, \ldots, \mu_{r}\right)$ is a set of system parameters, transfer functions $G_{1}(s ; \boldsymbol{\mu}), G_{2}(s ; \boldsymbol{\mu})$ have a low-pass characteristics and a polynomial-type nonlinear element $N[u]$ is a monotone increasing function of $u$.

We apply the HB method (cf. Appendix A) to the system Eq. (1). Thus, the HB equation is described by

$$
\boldsymbol{f}(\boldsymbol{x} ; \boldsymbol{\mu}, \boldsymbol{e}) \equiv\left(f_{0 \mathrm{r}}, f_{1 \mathrm{r}}, f_{1 \mathrm{~s}}, \ldots, f_{n \mathrm{r}}, f_{n \mathrm{~s}}\right)^{\mathrm{T}}=\mathbf{0} \in \mathbb{R}^{m} .
$$

where $\boldsymbol{x} \equiv\left(x_{0 \mathrm{r}}, x_{1 \mathrm{r}}, x_{1 \mathrm{~s}}, \ldots, x_{n \mathrm{r}}, x_{n \mathrm{~s}}\right)^{\mathrm{T}} \in \mathbb{R}^{m}$ is a vector of variables, $\boldsymbol{e} \equiv\left(e_{0 \mathrm{r}}, e_{1 \mathrm{r}}, e_{1 \mathrm{~s}}, \ldots, e_{n \mathrm{r}}, e_{n \mathrm{~s}}\right)^{\mathrm{T}} \in \mathbb{R}^{m}$ is a vector of frequency components of the input function, $(\cdot)^{\mathrm{T}}$ denote the transposition, $n$ is the number of specific frequency components, and $m=2 n+1$ denotes the number of unknowns.

\subsection{Error Bound for HB Method}

Because the HB method has a truncation error, we calculate guaranteed solutions evaluated by an error bound. The error bound is $m-1$ dimensional surface in $m$ dimensional space. In order to obtain the error bound, we extend the method reported in [9] to the periodically forced system (cf. Appendix $\mathrm{B})$. The error bound is calculated by removing the variable $\lambda$ from Eqs. (A. 16) and (A. 24).

However, the numerical computation of the error bound is very time-consuming compared with solving the $\mathrm{HB}$ equation because we need to express the high dimensional error bound using a set of numerical values.

\section{Algebraic Representation of Error Bound}

\subsection{Error Bound by Gröbner Base}

To overcome the difficulty of the numerical method, we try to represent the error bound algebraically using Gröbner base. In order to apply Gröbner techniques [16], [17], we transform Eqs. (A.16) and (A.24) into polynomial equations. Multiplying the both sides of Eq. (A. 16) by (1 $\lambda H)^{2 p}$, we rewrite Eq. (A. 16) to the following equation;

$$
\begin{aligned}
& f_{\mathrm{EB} 1}(\lambda, \boldsymbol{x} ; \boldsymbol{\mu}, \boldsymbol{e}) \equiv \lambda(1-\lambda H)^{2 p} \\
& -\sum_{i=0}^{p}(2 i+1) c_{2 i+1}(1-\lambda H)^{2(p-i)}\left(\sum_{k=0}^{n} \sqrt{x_{k \mathrm{r}}^{2}+x_{k \mathrm{~s}}^{2}}\right)^{2 i}=0 .
\end{aligned}
$$

Moreover, multiplying the both sides of Eq. (A. 24) by $1-$ $\lambda H$ and squaring it, we obtain

$$
\begin{aligned}
& f_{\mathrm{EB} 2}(\lambda, \boldsymbol{x} ; \boldsymbol{\mu}, \boldsymbol{e}) \\
& \quad \equiv \lambda^{4} H^{2} \sum_{k=0}^{n}\left(x_{k \mathrm{r}}^{2}+x_{k \mathrm{~s}}^{2}\right)-(1-\lambda H)^{2} \sum_{k=0}^{n}\left(f_{k \mathrm{r}}^{2}+f_{k \mathrm{~s}}^{2}\right)=0 .
\end{aligned}
$$

Equations (4) and (5) are polynomial equations with respect to $\lambda$.

Because Gröbner base of lexicographic order $\lambda>x_{k \mathrm{r}}$ (or $\lambda>x_{k \mathrm{~s}}$ ) enables to eliminate $\lambda$ from Eqs. (4) and (5). we can obtain the following algebraic representation of the error bound [10];

$$
g_{\mathrm{EB}}(\boldsymbol{x} ; \boldsymbol{\mu}, \boldsymbol{e})=0 .
$$

However, the computational cost of Gröbner base is highly dependent on the complexity of Eqs. (4) and (5). In particular, the computational cost increases exponentially according to the expansion of the number $n$. Thus, the algebraic representation (6) can not be calculated by the naive method in [10] if we consider more than 2 frequency components.

\subsection{Efficient Method to Obtain Algebraic Representation}

In order to resolve the problem of Gröbner base, we propose an efficient method to obtain the algebraic representation of the error bound using transformations of variables [11]. Because the number $n$ of the specific frequency components complicates only the norms in Eqs. (4) and (5), we transform the norms into new variables;

$$
\begin{aligned}
& \alpha(\boldsymbol{x}) \equiv\left\|u_{\mathrm{L}}(\tau)\right\|_{1}=\sum_{k=0}^{n} \sqrt{x_{k \mathrm{r}}^{2}+x_{k \mathrm{~s}}^{2}}, \\
& \beta(\boldsymbol{x}) \equiv\left\|u_{\mathrm{L}}(\tau)\right\|_{2}^{2}=\sum_{k=0}^{n}\left(x_{k \mathrm{r}}^{2}+x_{k \mathrm{r}}^{2}\right), \\
& \gamma(\boldsymbol{x} ; \boldsymbol{\mu}, \boldsymbol{e}) \equiv\left\|\mathrm{FH}\left(u_{\mathrm{L}}\right)\right\|_{2}{ }^{2} \\
& =\sum_{k=0}^{n}\left(f_{k \mathrm{r}}^{2}(\boldsymbol{x} ; \boldsymbol{\mu}, \boldsymbol{e})+f_{k \mathrm{~s}}^{2}(\boldsymbol{x} ; \boldsymbol{\mu}, \boldsymbol{e})\right) .
\end{aligned}
$$

Thus, using the transformation of the variable $\boldsymbol{x}$ into $\alpha, \beta, \gamma$, we rewrite Eqs. (4) and (5) by

$$
\begin{aligned}
& f_{\mathrm{EB} 1}(\lambda, \alpha ; H)=\lambda(1-\lambda H)^{2 p} \\
& \quad-\sum_{i=0}^{p}(2 i+1) c_{2 i+1}(1-\lambda H)^{2(p-i)} \alpha^{2 i}=0, \\
& f_{\mathrm{EB} 2}(\lambda, \beta, \gamma ; H)=\lambda^{4} H^{2} \beta-(1-\lambda H)^{2} \gamma=0 .
\end{aligned}
$$

Because the representations (10) and (11) have only 4 variables $\lambda, \alpha, \beta, \gamma$ instead of $m+1$ variables $\lambda, x_{0 \mathrm{r}}, x_{1 \mathrm{r}}$, $x_{1 \mathrm{~s}}, \ldots, x_{n \mathrm{~s}}$ in Eqs. (4) and (5), the computational cost of Gröbner base can be reduced.

Then, $g_{\mathrm{EB}}(\alpha, \beta, \gamma ; H)$ is obtained by the elimination of $\lambda$ using Gröbner base from Eqs. (10) and (11). Since the expressions of Eqs. (10) and (11) are far simpler than those of Eqs. (4) and (5), the computational cost of obtaining $g_{\mathrm{EB}}(\alpha, \beta, \gamma ; H)$ is remarkably less than the cost of Eq. (6) by the naive method. After we calculate $g_{\mathrm{EB}}(\alpha, \beta, \gamma ; H)$, the algebraic representation (6) is obtained by the substitutions of $\alpha, \beta, \gamma, H$ into $g_{\mathrm{EB}}(\alpha, \beta, \gamma ; H)$. Thus, the algorithm is given by

$\mathrm{S} 1$. We give the polynomial equations $f_{\mathrm{EB} 1}(\lambda, \alpha ; H)=0$ and $f_{\mathrm{EB} 2}(\lambda, \beta, \gamma ; H)=0$.

S2. We obtain $g_{\mathrm{EB}}(\alpha, \beta, \gamma ; H)$ by the elimination of $\lambda$ using Gröbner base of order $\lambda>(\alpha, \beta, \gamma)$ from $f_{\mathrm{EB} 1}$ and $f_{\mathrm{EB} 2}$. 
S3. We obtain the algebraic representation (6) of the error bound by the substitution of $\alpha, \beta, \gamma, H$ into $g_{\mathrm{EB}}(\alpha, \beta, \gamma ; H)$.

As is easily seen from this algorithm, we can obtain the algebraic representation (6) of the error bound even if we consider many frequency components. Moreover, the representation $g_{\mathrm{EB}}(\alpha, \beta, \gamma ; H)$ is uniquely determined only by the nonlinear element $N[u]$ because the transfer functions $G_{1}$ and $G_{2}$ are contained only in the variable $\gamma$ and the parameter $H$ in $g_{\mathrm{EB}}(\alpha, \beta, \gamma ; H)$ symbolically.

\subsection{Example}

We apply the proposed method to Duffing equation;

$$
\frac{\mathrm{d}^{2} u(\tau)}{\mathrm{d} \tau^{2}}+\mu \frac{\mathrm{d} u(\tau)}{\mathrm{d} \tau}+u^{3}=E \cos \tau .
$$

This equation can be rewritten as Eq. (13);

$$
\begin{aligned}
& u(\tau)=G_{1}(s ; \mu)\left\{v(\tau)-G_{2}(s ; \mu) N[u(\tau)]\right\}, \\
& v(\tau)=E \cos \tau, \quad N[u(\tau)]=u^{3}, \\
& G_{1}(s ; \mu)=\frac{1}{s^{2}+\mu s}, G_{2}=1, G=G_{1} .
\end{aligned}
$$

The equations $f_{\mathrm{EB} 1}$ and $f_{\mathrm{EB} 2}$ is written by

$$
\begin{aligned}
& f_{\mathrm{EB} 1}(\lambda, \alpha ; H)=\lambda(1-\lambda H)^{2}-3 \alpha^{2}=0, \\
& f_{\mathrm{EB} 2}(\lambda, \beta, \gamma ; H)=\lambda^{4} H^{2} \beta-(1-\lambda H)^{2} \gamma=0 .
\end{aligned}
$$

Thus, the following algebraic representation $g_{\mathrm{EB}}(\alpha, \beta, \gamma ; H)$ of the error bound is obtained by the elimination of $\lambda$ using Gröbner base of order $\lambda>(\alpha, \beta, \gamma)$;

$$
\begin{gathered}
g_{\mathrm{EB}}(\alpha, \beta, \gamma ; H)=9 \alpha^{4} H^{6} \gamma^{3}-135 \alpha^{4} \beta H^{4} \gamma^{2} \\
-6 \alpha^{2} \beta H^{3} \gamma^{2}-270 \alpha^{6} \beta^{2} H^{3} \gamma+225 \alpha^{4} \beta^{2} H^{2} \gamma \\
-30 \alpha^{2} \beta^{2} H \gamma+\beta^{2} \gamma-81 \alpha^{8} \beta^{3} H^{2}=0 .
\end{gathered}
$$

Let us compare the proposed method using Gröbner base of order $\lambda>\alpha>\beta>\gamma$ with the naive method using the order $\lambda>x_{1 \mathrm{r}}>x_{1 \mathrm{~s}}$. The computational cost of both methods is shown in Table 1 where $n=1$. From this table, we can confirm the efficiency of the proposed method.

Further, Eq. (16) does not contain the transfer function $G(s ; \mu)$ and the number of the specific frequency components explicitly. Thus, when we fix the nonlinear element $N[u]$, we can obtain the algebraic representation (6) from $g_{\mathrm{EB}}(\alpha, \beta, \gamma ; H)$ even if we consider many frequency components.

Table 1 Comparison of computational cost between proposed method and naive method $(n=1)$.

\begin{tabular}{c|ccc}
\hline Method & $\begin{array}{c}\text { Order of } \\
\text { variables }\end{array}$ & $\begin{array}{c}\text { Computation } \\
\text { time [s] }\end{array}$ & $\begin{array}{c}\text { Required } \\
\text { memory [MB] }\end{array}$ \\
\hline \hline Naive method & $\lambda>x_{1 \mathrm{r}}>x_{1 \mathrm{~s}}$ & 7425 & 956 \\
Proposed method & $\lambda>\alpha>\beta>\gamma$ & 0.007 & 1.09 \\
\hline
\end{tabular}

Calculated by a PC with Xeon $3.06 \mathrm{GHz} \mathrm{CPU}$.

\section{Fast Computation of Approximated Error Bound}

\subsection{Quadratic Approximation of Error Bound}

In order to visualize the high dimensional error bound, we project the error bound to a complex plane of a target frequency component. However, the computation of the projection is very time-consuming.

We propose a fast computational method to obtain an approximated projection of the error bound. The error bound is in a neighborhood of the solution of the HB equation, and resembles an ellipsoidal body. Thus, we approximate the error bound to the quadratic form using variations of the solution.

Let us consider a projection of the error bound to a complex plane $\left(x_{l \mathrm{r}}, x_{\mathrm{ls}}\right)$ for $l \in\{1, \ldots, n\}$. Then we rewrite the vector of the variable

$$
\begin{aligned}
\boldsymbol{x} & =\left(x_{l \mathrm{r}}, x_{l \mathrm{~s}}, x_{0 \mathrm{r}}, \ldots, x_{l-1 \mathrm{r}}, x_{l-1 \mathrm{~s}} x_{l+1 \mathrm{r}}, x_{l+1 \mathrm{~s}} \ldots, x_{n \mathrm{r}}, x_{n \mathrm{~s}}\right)^{\mathrm{T}} \\
& \equiv\left(x_{1}, x_{2}, \ldots, x_{m}\right)^{\mathrm{T}} .
\end{aligned}
$$

Further, we consider that the variable $\boldsymbol{x}$ is described by

$$
x=\hat{x}+\Delta x,
$$

where, $\hat{\boldsymbol{x}} \equiv\left(\hat{x}_{1}, \hat{x}_{2}, \ldots, \hat{x}_{m}\right)^{\mathrm{T}}$ is a vector of the solution of the HB equation and $\Delta \boldsymbol{x} \equiv\left(\Delta x_{1}, \Delta x_{2}, \ldots, \Delta x_{m}\right)^{\mathrm{T}}$ is a vector of its variations.

Using $\Delta \boldsymbol{x}$ and Taylor expansion, we obtain the quadratic approximation $g_{\triangle \mathrm{EB}}(\Delta \boldsymbol{x} ; \boldsymbol{\mu}, \boldsymbol{e})$ of the error bound as follows;

$$
\begin{aligned}
& g_{\mathrm{EB}}(\boldsymbol{x} ; \boldsymbol{\mu}, \boldsymbol{e}) \approx g_{\Delta \mathrm{EB}}(\Delta \boldsymbol{x} ; \boldsymbol{\mu}, \boldsymbol{e}) \\
& \quad=\sum_{i=1}^{m} a_{i i} \Delta x_{i}^{2}+2 \sum_{i=1}^{m} \sum_{j=i+1}^{m} a_{i j} \Delta x_{i} \Delta x_{j}+2 \sum_{i=1}^{m} a_{0 i} \Delta x_{i}+a_{00} .
\end{aligned}
$$

Then, the approximated error bound is rewritten by

$$
g_{\Delta \mathrm{EB}}(\Delta x ; \boldsymbol{\mu}, \boldsymbol{e})=\left(1, \Delta \boldsymbol{x}^{\mathrm{T}}\right) \boldsymbol{A}\left[\begin{array}{c}
1 \\
\Delta \boldsymbol{x}
\end{array}\right]=0,
$$

where

$$
\boldsymbol{A} \equiv \boldsymbol{A}(\hat{\boldsymbol{x}}, \boldsymbol{\mu}, \boldsymbol{e}) \equiv\left[\begin{array}{cccc}
a_{00} & a_{01} & \cdots & a_{0 m} \\
a_{01} & a_{11} & \cdots & a_{1 m} \\
\vdots & \vdots & \ddots & \vdots \\
a_{0 m} & a_{1 m} & \cdots & a_{m m}
\end{array}\right] \in \mathbb{R}^{(m+1) \times(m+1)} .
$$

In order to obtain the projection of the approximated error bound $g_{\triangle \mathrm{EB}}(\Delta \boldsymbol{x} ; \boldsymbol{\mu}, \boldsymbol{e})$, we decompose $\boldsymbol{A}(\hat{\boldsymbol{x}}, \boldsymbol{\mu}, \boldsymbol{e})$ and $\Delta \boldsymbol{x}$ into

$$
\boldsymbol{A} \equiv\left[\begin{array}{ll}
\boldsymbol{A}_{1} & \boldsymbol{A}_{2} \\
\boldsymbol{A}_{2}^{\mathrm{T}} & \boldsymbol{A}_{3}
\end{array}\right],\left[\begin{array}{c}
1 \\
\Delta \boldsymbol{x}
\end{array}\right] \equiv\left[\begin{array}{l}
\Delta \boldsymbol{x}_{1} \\
\Delta \boldsymbol{x}_{2}
\end{array}\right], \Delta \boldsymbol{x}_{1}=\left[\begin{array}{c}
1 \\
\Delta x_{1} \\
\Delta x_{2}
\end{array}\right], \Delta \boldsymbol{x}_{2}=\left[\begin{array}{c}
\Delta x_{3} \\
\vdots \\
\Delta x_{m}
\end{array}\right],
$$

where partial matrices of $\boldsymbol{A}$ denote $\boldsymbol{A}_{1} \in \mathbb{R}^{3 \times 3}, \boldsymbol{A}_{2} \in$ 
$\mathbb{R}^{(m-2) \times 3}$ and $\boldsymbol{A}_{3} \in \mathbb{R}^{(m-2) \times(m-2)}$, respectively. Now let

$$
\nabla g_{\Delta \mathrm{EB}}=\left(\frac{\partial g_{\Delta \mathrm{EB}}}{\partial \Delta x_{1}}, \ldots \frac{\partial g_{\Delta \mathrm{EB}}}{\partial \Delta x_{m}}\right)^{\mathrm{T}} \in \mathbb{R}^{m},
$$

be a gradient vector of $g_{\Delta \mathrm{EB}}$. Then, the boundary of the projected error bound to $\left(x_{1}, x_{2}\right)$ plane satisfies that $\nabla g_{\Delta \mathrm{EB}}$ is orthogonal to the following unit vectors which are parallel to the $x_{3}, \ldots, x_{m}$ axes.

$$
\begin{gathered}
(0,0,0,1,0, \ldots, 0)^{\mathrm{T}}, \\
(0,0,0,0,1, \ldots, 0)^{\mathrm{T}}, \\
\vdots \\
(0,0,0,0,0, \ldots, 1)^{\mathrm{T}} .
\end{gathered}
$$

Namely, the projection of $g_{\Delta \mathrm{EB}}(\Delta \boldsymbol{x})$ satisfies

$$
\frac{\partial g_{\Delta \mathrm{EB}}(\Delta \boldsymbol{x})}{\partial \Delta x_{k}}=0, k=3, \ldots, m .
$$

Thus, applying this relation to Eq. (20), we obtain a constraint for the projection;

$$
\boldsymbol{A}_{2}^{\mathrm{T}} \Delta \boldsymbol{x}_{1}+\boldsymbol{A}_{3} \Delta \boldsymbol{x}_{2}=\mathbf{0}
$$

As a result, the projection of the approximated error bound is represented by

$$
\begin{aligned}
\left(1, \Delta \boldsymbol{x}^{\mathrm{T}}\right) \boldsymbol{A}\left[\begin{array}{c}
1 \\
\Delta \boldsymbol{x}
\end{array}\right] & =\left(\Delta \boldsymbol{x}_{1}^{\mathrm{T}}, \Delta \boldsymbol{x}_{2}^{\mathrm{T}}\right)\left[\begin{array}{c}
\boldsymbol{A}_{1} \Delta \boldsymbol{x}_{1}+\boldsymbol{A}_{2} \Delta \boldsymbol{x}_{2} \\
\mathbf{0}
\end{array}\right] \\
& =\Delta \boldsymbol{x}_{1}^{\mathrm{T}} \boldsymbol{A}_{1} \Delta \boldsymbol{x}_{1}+\Delta \boldsymbol{x}_{1}^{\mathrm{T}} \boldsymbol{A}_{2} \Delta \boldsymbol{x}_{2} \\
& =\Delta \boldsymbol{x}_{1}^{\mathrm{T}}\left(\boldsymbol{A}_{1}-\boldsymbol{A}_{2} \boldsymbol{A}_{3}^{-1} \boldsymbol{A}_{2}^{\mathrm{T}}\right) \Delta \boldsymbol{x}_{1}=0 .
\end{aligned}
$$

Finally, the substitution of $\Delta x_{1}=x_{1}-\hat{x}_{1}, \Delta x_{2}=x_{2}-\hat{x}_{2}$ into Eq. (26) gives the approximated projection of the error bound.

The quadratic approximation algorithm is written by

$\mathrm{S} 1$. We calculate the algebraic representation (6) of the error bound using Gröbner base.

$\mathrm{S} 2$. We set the target complex plane $\left(x_{1}, x_{2}\right)$ and other variables $x_{3}, \ldots, x_{m}$.

S3. We obtain algebraic representations of the elements $a_{i j}(\hat{\boldsymbol{x}}, \boldsymbol{\mu}, \boldsymbol{e}),(i, j=0, \ldots, m, i \leq j)$ of the matrix $\boldsymbol{A}$ with the solution $\hat{\boldsymbol{x}}$ of the HB equation and the system parameters $\boldsymbol{\mu}, \boldsymbol{e}$.

S4. We determine $a_{i j}$ by the substitution of the given solution $\hat{\boldsymbol{x}}$ and parameters $\boldsymbol{\mu}, \boldsymbol{e}$ into $a_{i j}(\hat{\boldsymbol{x}}, \boldsymbol{\mu}, \boldsymbol{e}),(i, j=$ $0, \ldots, m, i \leq j)$.

S5. We obtain the projection of the approximated error bound by $\boldsymbol{A}_{1}-\boldsymbol{A}_{2} \boldsymbol{A}_{3}^{-1} \boldsymbol{A}_{2}^{\mathrm{T}}$ and the substitution of $\Delta x_{1}=$ $x_{1}-\hat{x}_{1}, \Delta x_{2}=x_{2}-\hat{x}_{2}$ into Eq. (26).

Although the proposed error bound approximately guarantees solutions of the HB equation, the projection of the error bound can be plotted easily on a two-dimensional space by this algorithm.

\subsection{Example}

We apply the quadratic approximation of the error bound to Duffing Eq. (12) where we assume that zero frequency components are zero for simplicity. We consider the approximated projection to fundamental frequency component, namely, $x_{1}=x_{1 \mathrm{r}}, x_{2}=x_{1 \mathrm{~s}}, x_{3}=x_{2 \mathrm{r}}, \ldots, x_{m-1}=$ $x_{n \mathrm{r}}, x_{m}=x_{n \mathrm{~s}}$, and $f_{1}=f_{1 \mathrm{r}}, f_{2}=f_{1 \mathrm{~s}}, f_{3}=f_{2 \mathrm{r}}, \ldots, f_{m-1}=$ $f_{n \mathrm{r}}, f_{m}=f_{n \mathrm{~s}}$, where $m=2 n$. Let the solution of the HB equation $\boldsymbol{f}(\boldsymbol{x} ; \mu, E)=\mathbf{0}$ be $\hat{\boldsymbol{x}}=\left(\hat{x}_{1}, \ldots, \hat{x}_{m}\right)^{\mathrm{T}}$. Then the matrix $\boldsymbol{A}$ of the approximated error bound is represented by the elements

$$
\begin{aligned}
& a_{00}=-81 H^{2} \hat{\alpha}_{0}^{8} \hat{\beta}_{0}, \\
& a_{0 i}=-81 H^{2} \hat{\alpha}_{0}^{8} \hat{x}_{i}+4 \hat{\alpha}_{0}^{7} \hat{\alpha}_{0 i} \hat{\beta}_{0}, \\
& a_{i i}=-81 H^{2} \hat{\alpha}_{0}^{6}\left(16 \hat{\alpha}_{0} \hat{\alpha}_{0 i} \hat{x}_{i}+8 \hat{\alpha}_{0} \hat{\alpha}_{i i} \hat{\beta}_{0}+28 \hat{\alpha}_{0 i}^{2} \hat{\beta}_{0}+\hat{\alpha}_{0}^{2}\right) \\
& \quad\left(225 \hat{\alpha}_{0}^{4} H^{2}-30 \hat{\alpha}_{0}^{4} H-270 \hat{\alpha}_{0}^{6} H^{3}+1\right) \sum_{k=1}^{m}\left(\frac{\partial f_{k}(\hat{\boldsymbol{x}} ; \mu, E)}{\partial \hat{x}_{i}}\right)^{2} \\
& a_{i j}=-81 H^{2} \hat{\alpha}_{0}^{6}\left(8 \hat{\alpha}_{0} \hat{\alpha}_{0 i} \hat{x}_{j}+8 \hat{\alpha}_{0} \hat{\alpha}_{0 j} \hat{x}_{i}+28 \hat{\alpha}_{0 i} \hat{\alpha}_{0 j} \hat{\beta}_{0}\right. \\
& \left.+4 \hat{\alpha}_{0} \hat{\alpha}_{i j} \hat{\beta}_{0}\right)+\left(225 \hat{\alpha}_{0}^{4} H^{2}-30 \hat{\alpha}_{0}^{4} H-270 \hat{\alpha}_{0}^{6} H^{3}+1\right) \\
& \sum_{k=1}^{m}\left(\frac{\partial f_{k}(\hat{\boldsymbol{x}} ; \mu, E)}{\partial \hat{x}_{j}} \frac{\partial f_{k}(\hat{\boldsymbol{x}} ; \mu, E)}{\partial \hat{x}_{i}}\right)
\end{aligned}
$$

where

$\hat{\alpha}_{0}=\sum_{k=1}^{n} \sqrt{\hat{x}_{2 k}^{2}+\hat{x}_{2 k+1}^{2}}, \hat{\alpha}_{0 i}=\frac{\hat{x}_{i}}{\hat{\alpha}_{i}}, \hat{\alpha}_{i i}=\frac{\hat{x}_{i}}{2 \hat{\alpha}_{i}}-\frac{\hat{x}_{i}^{2}}{2 \hat{\alpha}_{i}^{3}}$,

$\hat{\alpha}_{i j}=\left\{\begin{array}{cc}-\frac{\hat{x}_{i} \hat{x}_{j}}{2 \hat{\alpha}_{i}} & |i-j|=1 \\ 0 & |i-j| \neq 1\end{array}, \hat{\alpha}_{i}=\left\{\begin{array}{cc}\sqrt{\hat{x}_{i}^{2}+\hat{x}_{i+1}^{2}} & i=1 \bmod 2 \\ \sqrt{\hat{x}_{i-1}^{2}+\hat{x}_{i}^{2}} & i=0 \bmod 2\end{array}\right.\right.$,

$\hat{\beta}_{0}=\sum_{k=1}^{m} \hat{x}_{k}^{2}, H=\frac{1}{(n+1) \sqrt{(n+1)^{2}+\mu^{2}}}$,

$i=1, \ldots, m, j=2, \ldots, m, i<j$.

In order to confirm the validity of the approximation, the projections by the proposed method and the method in [9] are shown in Fig. 2 where $\mu=0.1, E=0.35, n=4,6,8$. We can see that the projection of the approximated error bound is very close to the projection in [9].

Moreover, the projection of the approximated error bound with the parameter $E$ varying from 0.1 to 0.4 is shown in Fig. 3 where $\mu=0.1$ and $n=4$. Because the elements (27), (28), (29) and (30) contain the system parameters symbolically, the approximated error bound can be easily obtained even if we change the system parameters as shown in Fig. 3.

Further, the computational time of the proposed method and the method in [9] for $n=4,6,8,20$ is shown in Table 2 when we vary the parameters $\mu$ from 0.1 to 1.0 , $E$ from 0.1 to 0.4 . Additionally, we also show the solving time of the HB equation in Table 2. Although the proposed method in Table 2 does not contain the computational cost of $g_{\mathrm{EB}}(\boldsymbol{x} ; \mu, E), g_{\mathrm{EB}}(\boldsymbol{x} ; \mu, E)$ is calculated only once and the 


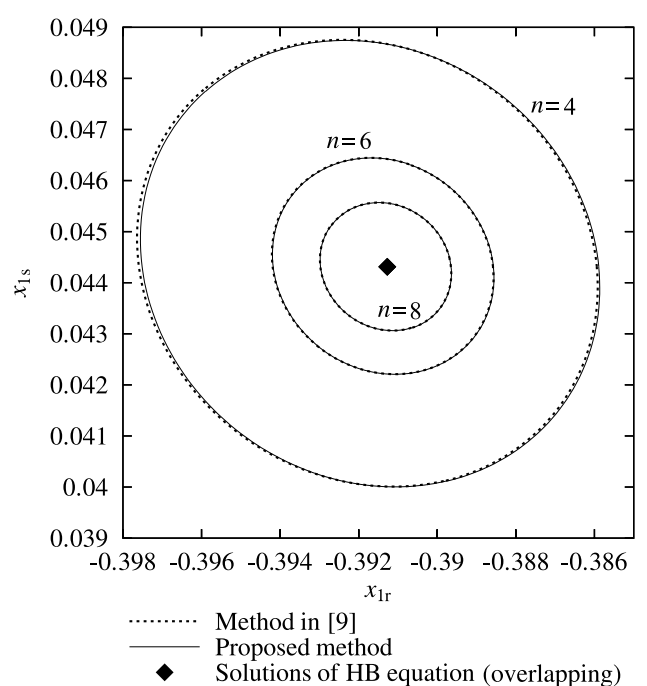

Fig. 2 Projections of error bound for HB method on the $\left(x_{1 \mathrm{r}}, x_{1 \mathrm{~s}}\right)$ plane $(\mu=0.1, E=0.35$ and $n=4,6,8)$.

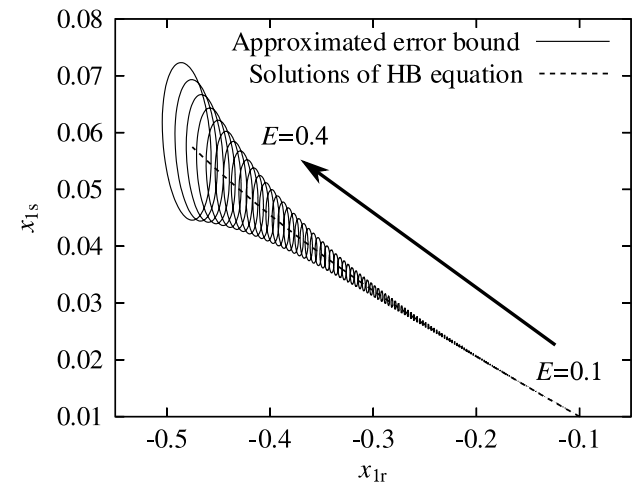

Fig. 3 Projection of approximated error bound with parameter $E$ varying from 0.1 to $0.4(\mu=0.1$ and $n=4)$.

Table 2 Computational time of projection of error bound [s] ( $\mu$ varied from 0.1 to 1.0 and $E$ varied from 0.1 to 0.4 , using Newton method with 90 $\times 300 \times 32$ points).

\begin{tabular}{c|rrrr}
\hline Method & $n=4$ & $n=6$ & $n=8$ & $n=20$ \\
\hline \hline HB method & 1.50 & 2.23 & 3.47 & 16.63 \\
\hline Method in [9] & 237.02 & 303.73 & 390.45 & 1172.47 \\
Proposed method & 7.52 & 8.70 & 10.29 & 26.22 \\
\hline Calculated by a PC with Xeon 3.06 GHz CPU.
\end{tabular}

computational cost is very low as shown in Table 1 . Thus, we can confirm that the proposed method reduces the computational cost of the error bound dramatically. Although the conventional method is very time-consuming compared with solving the HB equation, the proposed method approximately guarantees the solutions as fast as solving the $\mathrm{HB}$ equation.

\section{Break Point of Error Bound}

\subsection{Break Point and Singular Point of Error Bound}

When we set the system parameters close to the bifurcation

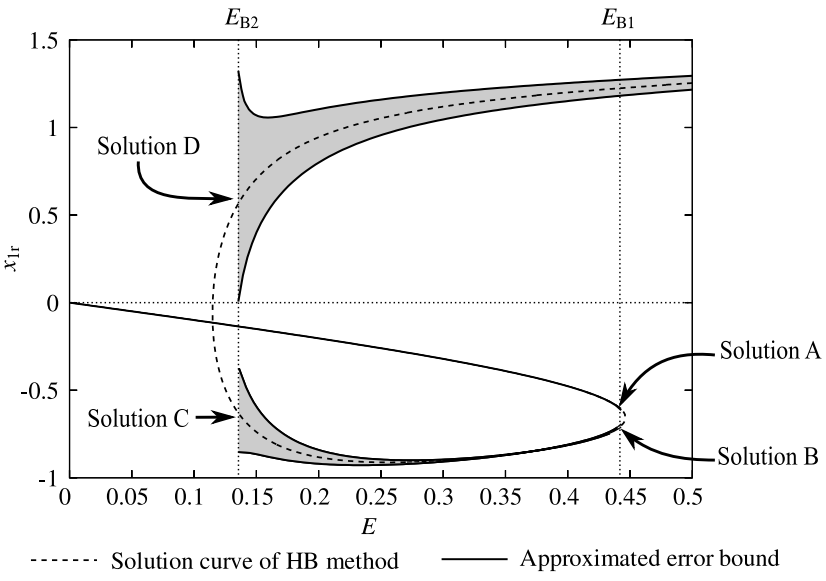

Fig. 4 Bifurcation diagram of HB method and approximated error bound $(\mu=0.1$ and $n=30)$.

parameters, there exist two solutions of the HB equation in a neighborhood. In such cases, two error bounds containing these solutions are broken by a collision of each other, and we can not hence guarantee the solutions near the bifurcation point. Let us call this collision point a break point of the error bound. We propose a method to obtain accurate break points using the algebraic representation of the error bound.

Because a gradient vector of singular points equals zero in general [16], [17], the break point of the error bound satisfies the following relations based on the algebraic representation (6)

$$
\left\{\begin{array}{rl}
g_{\mathrm{EB}}(\boldsymbol{x} ; \boldsymbol{\mu}, \boldsymbol{e}) & =0 \\
\nabla g_{\mathrm{EB}}(\boldsymbol{x} ; \boldsymbol{\mu}, \boldsymbol{e}) & =\mathbf{0}
\end{array},\right.
$$

where a gradient vector $\nabla g_{\mathrm{EB}}(\boldsymbol{x} ; \boldsymbol{\mu}, \boldsymbol{e})$ is written by

$$
\nabla g_{\mathrm{EB}}=\left(\frac{\partial g_{\mathrm{EB}}}{\partial x_{0 \mathrm{r}}}, \frac{\partial g_{\mathrm{EB}}}{\partial x_{1 \mathrm{r}}}, \frac{\partial g_{\mathrm{EB}}}{\partial x_{1 \mathrm{~s}}}, \ldots \frac{\partial g_{\mathrm{EB}}}{\partial x_{n \mathrm{r}}}, \frac{\partial g_{\mathrm{EB}}}{\partial x_{n \mathrm{~s}}}\right)^{\mathrm{T}} \in \mathbb{R}^{m}
$$

Thus, if we view one system parameter $\varepsilon \in\{\boldsymbol{\mu}, \boldsymbol{e}\}$ as the variable, the simultaneous Eq. (31) gives the break point $(\boldsymbol{x}, \boldsymbol{\varepsilon})$. We obtain the break point of the error bound by numerical method using an initial point $\left(\left(\hat{\boldsymbol{x}}_{1}+\hat{\boldsymbol{x}}_{2}\right) / 2, \hat{\varepsilon}\right)$, where $\hat{\varepsilon}$ is the parameter value close to the bifurcation parameters, and $\hat{\boldsymbol{x}}_{1}, \hat{\boldsymbol{x}}_{2}$ denote two close numerical solutions of the HB equation $f(x ; \hat{\varepsilon})=\mathbf{0}$.

The algorithm to obtain the break point of the error bound is described by

S1. We calculate the algebraic representation (6) of the error bound using Gröbner base.

$\mathrm{S} 2$. We select the parameter $\varepsilon$ in the system parameters $\boldsymbol{\mu}, \boldsymbol{e}$.

S3. Using the initial value $\left(\left(\hat{\boldsymbol{x}}_{1}+\hat{\boldsymbol{x}}_{2}\right) / 2, \hat{\varepsilon}\right)$, we obtain the break point of the error bound by solving Eq. (31).

\subsection{Example}

We obtain the break points of the error bound for Duffing Eq. (12), where we assume that zero frequency component is neglected for simplicity. Let us select the parameter $\varepsilon=E$ 


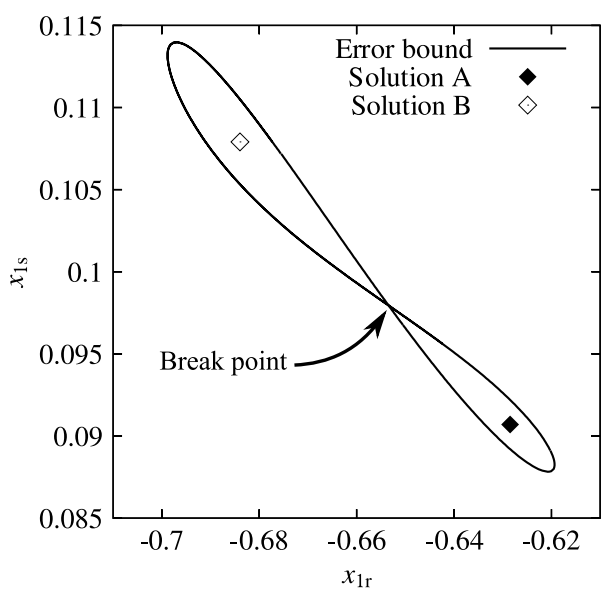

Fig. 5 Projection $\left(x_{1 \mathrm{r}}, x_{1 \mathrm{~s}}\right)$ of error bound at parameter $E_{\mathrm{B} 1}$ of break point $\left(E_{\mathrm{B} 1}=0.445168, \mu=0.1\right.$ and $\left.n=30\right)$.

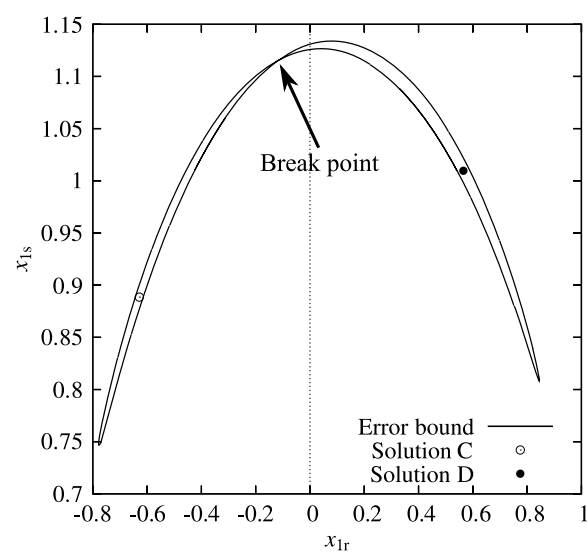

Fig. 6 Projection $\left(x_{1 \mathrm{r}}, x_{1 \mathrm{~s}}\right)$ of error bound at parameter $E_{\mathrm{B} 2}$ of break point $\left(E_{\mathrm{B} 2}=0.135366, \mu=0.1\right.$ and $\left.n=30\right)$.

and let $\mu=0.1, n=30$. Then the bifurcation diagram $E-x_{1 \mathrm{r}}$ is shown in Fig. 4. Additionally, we also show the approximated error bound in Fig. 4. Namely, we can guarantee the solutions of the HB equation in a gray region. Thus, the bifurcation points in Fig. 4 lie close to $E=0.45$ and $E=0.12$.

Using the proposed method, we can calculate the parameters $E_{\mathrm{B} 1}=0.445168$ and $E_{\mathrm{B} 2}=0.135366$ of the break points. We show the projection of the error bound at $E_{\mathrm{B} 1}$ and $E_{\mathrm{B} 2}$ in Fig. 5 and Fig. 6, respectively. The Solution A, B, C and D in Fig. 5 and Fig. 6 correspond to the Solution A, B, C and D in Fig. 4. Thus, we can confirm that the proposed method enables to obtain the accurate break points of the error bound by the collisions.

\section{Conclusion}

We proposed an algebraic representation of an error bound for HB method using Gröbner base. Further, we proposed an efficient method to calculate the algebraic representation using transformations of variables. The proposed method does not depend on linear elements of the system and the number of specific frequency components. Next, we proposed a fast computational method of an approximated error bound by a quadratic approximation using the algebraic representation. We confirmed that the quadratic approximation guarantees approximately the solutions as fast as solving the HB equation. Moreover, we proposed a method to obtain accurate break points of the error bound near bifurcation points. In this way, algebraic approach is very powerful for high dimensional varieties such as error bounds.

\section{Acknowledgment}

This work was supported by the 21st Century COE Program 14213201 and the GCOE Program.

\section{References}

[1] C. Hayashi, Nonlinear oscillations in physical systems, McGrawHill, New York, 1964.

[2] A.I. Mees, Dynamics of feedback systems, Wiley-Interscience, New York, 1981

[3] M. Basso, R. Genesio, and A. Tesi, "A frequency method for predicting limit cycle bifurcations," Nonlinear Dynamics, vol.13, no.4 pp.339-360, Aug. 1997.

[4] C. Piccardi, "Bifurcations of limit cycles in periodically forced nonlinear systems: The harmonic balance approach," IEEE Trans. Circuits Syst. I, vol.41, no.4, pp.315-320, April 1994.

[5] J.L. Moiola and G. Chen, Hopf Bifurcation Analysis: A Frequency Domain Approach, Series on Nonlinear Science, Series A, vol.21, World Scientific, Singapore, 1996.

[6] F. Bonani, and M. Gilli, "Analysis of stability and bifurcations of limit cycles in Chua's circuit through the harmonic balance approach," IEEE Trans. Circuits Syst. I, vol.46, no.8, pp.881-890, Aug. 1999.

[7] A.R. Bergen and R.L. Franks, "Justification of the describing function method," SIAM J. Control Optim., vol.9, pp.568-569, 1971.

[8] A.I. Mees and A.P. Bergen, "Describing functions revisited," IEEE Trans. Autom. Control, vol.AC-20, no.4, pp.473-478, 1975.

[9] F.L. Swern, "Analysis of oscillations in systems with polynomialtype nonlinearities using describing functions," IEEE Trans. Autom. Control, vol.AC-28, no.1, pp.31-41, 1983.

[10] M. Yagi, T. Hisakado, and K. Okumura, "Algebraic representation of error bounds for describing function using Gröebner base," Proc. ISCAS2005, pp.2831-2834, 2005.

[11] M. Yagi and T. Hisakado, "Fast computation of approximated error bound for harmonic balance method using algebraic representation," Proc. NOLTA2007, pp.250-253, Sept. 2007.

[12] K. Okumura, "Classifying nonlinear circuits by Gröbner base," Proc. NDES98, pp.267-270, July 1998.

[13] T. Hisakado and K. Okumura, "Mode decomposition of global bifurcation diagram with Gröbner bases," Phys. Lett. A, vol.292, pp.263268, Jan. 2002.

[14] M. Yagi, T. Hisakado, and K. Okumura, "Decomposition of bifurcation diagram for periodic oscillation using ideal quotient," Proc. NOLTA2005, pp.34-37, Oct. 2005.

[15] M. Yagi, T. Hisakado, and K. Okumura, "Efficient applications of invariants to harmonic balance equation using Gröbner base," IEICE Trans. Fundamentals, vol.E90-A, no.10, pp.2178-2186, Oct. 2007.

[16] D. Cox, J. Little, and D. O'shea, Ideals, Varieties, and Algorithms, Springer-Verlag, New York, 1992.

[17] D. Cox, J. Little, and D. O'shea, Using Algebraic Geometry, Springer-Verlag, New York, 1998.

[18] J.M. Ortega and W.C. Rheinboldt, Iterative Solution of Nonlinear Equations in Several Variables, Academic Press, New York, 1970

[19] C.B. Garcia, "Computation of solutions to nonlinear equations under 
homotopy invariance," Mathematics of Operations Research, vol.2, no.1, pp.25-29, Feb. 1977.

[20] M.H. Shih, "Bolzano's theorem in several complex variables," Proc. Amcr. Math. Soc., vol.79, no.1, pp.32-34, May 1980.

\section{Appendix A: Derivation of HB Equation}

Let us apply the HB method to the system equation (1). We assume that Eq. (1) has a periodic solution with the period $2 \pi$. Thus, the solution $u(\tau)$ is given by

$$
u(\tau) \equiv \sum_{k=0}^{\infty} \mathfrak{R}\left[x_{k}^{*} e^{\mathrm{j} k \tau}\right]=\sum_{k=0}^{\infty} \mathfrak{R}\left[\left(x_{k \mathrm{r}}+\mathrm{j} x_{k \mathrm{~s}}\right) e^{\mathrm{j} k \tau}\right],
$$

where $x_{k}^{*} \in \mathbb{C}, x_{k \mathrm{r}}, x_{k \mathrm{~s}} \in \mathbb{R}$ for $k=0, \ldots, n, \mathbb{C}$ is a set of complex numbers, $\mathbb{R}$ is a set of real numbers, $x_{0 \mathrm{~s}}=0$ and $\mathfrak{R}[\cdot]$ denotes the real part. Now, assuming that a projection operator $K_{\mathrm{L}}$ expresses the truncation of Fourier series, we approximate the above solution by

$$
\begin{aligned}
u_{\mathrm{L}}(\tau) \equiv K_{\mathrm{L}} u(\tau) & \equiv \sum_{k=0}^{n} \mathfrak{R}\left[x_{k}^{*} e^{\mathrm{j} k \tau}\right] \\
& =\sum_{k=0}^{n} \mathfrak{R}\left[\left(x_{k \mathrm{r}}+\mathrm{j} x_{k \mathrm{~s}}\right) e^{\mathrm{j} k \tau}\right],
\end{aligned}
$$

Using the operator $K_{\mathrm{L}}$ and approximated solution (A.2), we rewrite Eq. (1) to

$\sum_{k=0}^{n} \mathfrak{R}\left[\left(x_{k}^{*}-G_{1}(\mathrm{j} k ; \boldsymbol{\mu})\left\{\left(e_{k}^{*}-G_{2}(\mathrm{j} k ; \boldsymbol{\mu}) y_{k}^{*}\right\}\right) e^{\mathrm{j} k \tau}\right]=0\right.$,

due to $s^{n} e^{\mathrm{j} \tau}=(\mathrm{j} k)^{n} e^{\mathrm{j} \tau}$, where

$$
K_{\mathrm{L}} v(\tau)=\sum_{k=0}^{n} e_{k}^{*} e^{\mathrm{j} k \tau}, \quad K_{\mathrm{L}} N\left[u_{\mathrm{L}}(\tau)\right]=\sum_{k=0}^{n} y_{k}^{*} e^{\mathrm{j} k \tau} .
$$

By this relation, $\mathrm{HB}$ equation is written by

$$
\begin{aligned}
& \boldsymbol{f}(\boldsymbol{x} ; \boldsymbol{\mu}, \boldsymbol{e}) \equiv\left(f_{0 \mathrm{r}}, f_{1 \mathrm{r}}, f_{1 \mathrm{~s}}, \ldots, f_{n \mathrm{r}}, f_{n \mathrm{~s}}\right)^{\mathrm{T}}=\mathbf{0} \in \mathbb{R}^{m}, \\
& f_{0 \mathrm{r}} \equiv \mathfrak{R}\left[x_{0}^{*}-G_{1}(0 ; \boldsymbol{\mu})\left\{e_{0}^{*}-G_{2}(0 ; \boldsymbol{\mu}) y_{0}^{*}\right\}\right], \\
& f_{k \mathrm{r}} \equiv \mathfrak{R}\left[x_{k}^{*}-G_{1}(\mathrm{j} k ; \boldsymbol{\mu})\left\{e_{k}^{*}-G_{2}(\mathrm{j} k ; \boldsymbol{\mu}) y_{k}^{*}\right\}\right], \\
& f_{k \mathrm{~s}} \equiv \mathfrak{J}\left[x_{k}^{*}-G_{1}(\mathrm{j} k ; \boldsymbol{\mu})\left\{e_{k}^{*}-G_{2}(\mathrm{j} k ; \boldsymbol{\mu}) y_{k}^{*}\right\}\right] \\
& \boldsymbol{x} \equiv\left(x_{0 \mathrm{r}}, x_{1 \mathrm{r}}, x_{1 \mathrm{~s}}, \ldots, x_{n \mathrm{r}}, x_{n \mathrm{~s}}\right)^{\mathrm{T}} \in \mathbb{R}^{m}, \\
& \boldsymbol{e} \equiv\left(e_{0 \mathrm{r}}, e_{1 \mathrm{r}}, e_{1 \mathrm{~s}}, \ldots, e_{n \mathrm{r}}, e_{n \mathrm{~s}}\right)^{\mathrm{T}} \in \mathbb{R}^{m}, \\
& e_{k}^{*}=e_{k \mathrm{r}}+\mathrm{j} e_{k \mathrm{~s}}, e_{0}^{*}=e_{0 \mathrm{r}}, \\
& k=1, \ldots, n,
\end{aligned}
$$

where $\mathfrak{I}[\cdot]$ denotes the imaginary.

\section{Appendix B: Error Bound of HB Method for Periodi- cally Forced System}

\section{B.1 Definition for Error Bound}

Let a projection operator $K_{\mathrm{H}}$ be

$$
\begin{aligned}
& u_{\mathrm{H}}(\tau)=K_{\mathrm{H}} u(\tau)=\sum_{k=n+1}^{\infty} \mathfrak{R}\left[\left(x_{k \mathrm{r}}+\mathrm{j} x_{k \mathrm{~s}}\right) e^{\mathrm{j} k \tau}\right], \\
& u(\tau)=u_{\mathrm{L}}(\tau)+u_{\mathrm{H}}(\tau), \quad K_{\mathrm{L}}+K_{\mathrm{H}}=I,
\end{aligned}
$$

where $I$ is an identity operator. Then, we define norms by

$l^{i}$ norm : $\|u(\tau)\|_{i} \equiv\left\{\sum_{k=0}^{\infty}\left(\sqrt{x_{k \mathrm{r}}^{2}+x_{k \mathrm{~s}}^{2}}\right)^{i}\right\}^{1 / i}$ for $i=1,2$,

$L^{\infty} \operatorname{norm}:\|u(\tau)\|_{\infty} \equiv \sup _{\tau \in[0,2 \pi)}|u(\tau)|$.

where

$$
\begin{aligned}
& \left\|u_{\mathrm{L}}(\tau)\right\|_{i} \equiv\left\{\sum_{k=0}^{n}\left(\sqrt{x_{k \mathrm{r}}^{2}+x_{k \mathrm{~s}}^{2}}\right)^{i}\right\}^{1 / i}, \\
& \left\|u_{\mathrm{H}}(\tau)\right\|_{i} \equiv\left\{\sum_{k=n+1}^{\infty}\left(\sqrt{x_{k \mathrm{r}}^{2}+x_{k \mathrm{~s}}^{2}}\right)^{i}\right\}^{1 / i} \text { for } i=1,2 .
\end{aligned}
$$

The $l^{1}$ norm satisfy $\|u(t)\|_{\infty} \leq\|u(t)\|_{1}$.

\section{B.2 Estimation of High Frequency Components}

We estimate the high frequency components. Let $\lambda$ be any positive number satisfying

$$
\lambda \geq\left\|\frac{\mathrm{d} N[u]}{\mathrm{d} u}\right\|_{\infty} .
$$

Applying the operator $K_{\mathrm{H}}$ to Eq. (1), we obtain the relation;

$$
u_{\mathrm{H}}(\tau)=-K_{\mathrm{H}} G(s ; \boldsymbol{\mu}) N\left[u_{\mathrm{L}}(\tau)+u_{\mathrm{H}}(\tau)\right]
$$

If

$$
\lambda H \equiv \lambda \sup _{k>n}|G(\mathrm{j} k ; \boldsymbol{\mu})|<1,
$$

is satisfied where $H(\boldsymbol{\mu})=\sup _{k>n}|G(\mathrm{j} k ; \boldsymbol{\mu})| \in \mathbb{R}$ is a constant, then there exists a unique $u_{\mathrm{H}}[9]$.

Using the contraction mapping theorem and the mean value theorem, we obtain the following relations;

$$
\left\|u_{\mathrm{H}}\right\|_{i} \leq \frac{\lambda H}{1-\lambda H}\left\|u_{\mathrm{L}}\right\|_{i} \text { for } i=1,2 .
$$

The inequality estimates the high frequency components by the low frequency components for $l^{1}$ and $l^{2}$ norms.

\section{B.3 Determination of $\lambda$}

The estimation of the high frequency components (A-14) gives the following relation;

$$
\|u\|_{1} \leq\left\|u_{\mathrm{L}}\right\|_{1}+\left\|u_{\mathrm{H}}\right\|_{1} \leq\left(1+\frac{\lambda H}{1-\lambda H}\right)\left\|u_{\mathrm{L}}\right\|_{1} .
$$

Thus, if we determine the variable $\lambda$ as

$$
\begin{aligned}
\lambda & =\sum_{i=0}^{p}(2 i+1) c_{2 i+1}\left(1+\frac{\lambda H}{1-\lambda H}\right)^{2 i}\left\|u_{\mathrm{L}}\right\|_{1}^{2 i} \\
& \geq \sum_{i=0}^{p}(2 i+1) c_{2 i+1}\|u\|_{1}^{2 i}=\left\|\frac{\mathrm{d} N[u]}{\mathrm{d} u}\right\|_{1} \\
& \geq\left\|\frac{\mathrm{d} N[u]}{\mathrm{d} u}\right\|_{\infty},
\end{aligned}
$$

then $\lambda$ satisfies Eq. (A. 11). 


\section{B.4 Error Bound by Homotopy Invariance}

Applying the operator $K_{\mathrm{L}}$ to Eq. (1), we obtain

$$
\begin{aligned}
\mathrm{FT}\left(u_{\mathrm{L}}\right) & \equiv G^{-1}(s ; \boldsymbol{\mu}) u_{\mathrm{L}}-\left\{G_{2}^{-1}(s ; \boldsymbol{\mu}) v(\tau)-K_{\mathrm{L}} N\left[u_{\mathrm{L}}+u_{\mathrm{H}}\right]\right\} \\
& =0 .
\end{aligned}
$$

Equation $(\mathrm{A} \cdot 18)$ corresponds to the low frequency components of Eq. (1), and $u_{\mathrm{L}}$ in Eq. (A. 18) is the exact solution of Eq. (1). Now, if we set $u_{\mathrm{H}}=0$ in Eq. (A. 18), then we obtain

$$
\begin{aligned}
\mathrm{FH}\left(u_{\mathrm{L}}\right) & \equiv G^{-1}(s ; \boldsymbol{\mu}) u_{\mathrm{L}}-\left\{G_{2}^{-1}(s ; \boldsymbol{\mu}) v(\tau)-K_{\mathrm{L}} N\left[u_{\mathrm{L}}\right]\right\} \\
& =0
\end{aligned}
$$

which corresponds to the HB Eq. (A.5). That is, $u_{\mathrm{L}}$ in Eq. (A.19) is an approximated solution by the HB Eq. $(\mathrm{A} \cdot 5)$.

In order to obtain the error bound from Eqs. (A. 18) and (A. 19), we use the following lemma of the homotopy invariance theorem [18]-[20].

Lemma 1. Let $\Omega$ be an open bounded set in $\mathbb{R}^{m}$ and let $\boldsymbol{f}, \boldsymbol{g}: \bar{\Omega} \rightarrow \mathbb{R}^{m}$ be two continuous maps where $\bar{\Omega}$ denotes the closure of the set $\Omega$. Let $\boldsymbol{y} \in \mathbb{R}^{m}$ be a certain vector. Suppose further that $\varepsilon$ satisfies $0<\varepsilon=\min \left\{\|\boldsymbol{f}(\boldsymbol{z})-\boldsymbol{y}\|_{2} \mid \boldsymbol{z} \in \partial \Omega\right\}$ where $\partial \Omega$ is the boundary of the set $\Omega$. If

$$
\|\boldsymbol{f}(\boldsymbol{z})-\boldsymbol{g}(\boldsymbol{z})\|_{2}<\boldsymbol{\varepsilon} \quad \forall \boldsymbol{z} \in \partial \Omega,
$$

then $\operatorname{deg}(\boldsymbol{f}, \Omega, \boldsymbol{y})=\operatorname{deg}(\boldsymbol{g}, \Omega, \boldsymbol{y})$ where we denote by $\operatorname{deg}(\boldsymbol{f}, \Omega, \boldsymbol{y})$ the degree of $\boldsymbol{f}$ with respect to $\Omega$ at $\boldsymbol{y}$.

If we set $\boldsymbol{f}(\boldsymbol{z})=\mathrm{FH}, \boldsymbol{g}(\boldsymbol{z})=\mathrm{FT}, \boldsymbol{z}=\boldsymbol{x}, \boldsymbol{y}=0$ in Eq. (A. 20), then $\operatorname{deg}(\mathrm{FH}, \Omega, 0)=\operatorname{deg}(\mathrm{FT}, \Omega, 0)$ is satisfied. Namely, if there exists a region $\Omega$ containing a single solution of Eq. (A. 19) on whose boundary

$$
\left\|\mathrm{FT}\left(u_{\mathrm{L}}\right)-\mathrm{FH}\left(u_{\mathrm{L}}\right)\right\|_{2}<\left\|\mathrm{FH}\left(u_{\mathrm{L}}\right)\right\|_{2}
$$

holds, then a solution of Eq. (A - 18) exists belonging to $\Omega$.

Using the mean value theorem and the estimation of the high frequency components $(\mathrm{A} \cdot 14)$, we obtain the following relation from Eq. $(\mathrm{A} \cdot 21)$;

$$
\begin{aligned}
\left\|\mathrm{FT}\left(u_{\mathrm{L}}\right)-\mathrm{FH}\left(u_{\mathrm{L}}\right)\right\|_{2} & =\left\|K_{\mathrm{L}} N\left[u_{\mathrm{L}}\right]-K_{\mathrm{L}} N\left[u_{\mathrm{L}}+u_{\mathrm{H}}\right]\right\|_{2} \\
& \leq \lambda\left\|u_{\mathrm{H}}\right\|_{2} \leq \frac{\lambda^{2} H}{1-\lambda H}\left\|u_{\mathrm{L}}\right\|_{2} .
\end{aligned}
$$

Because inequality

$$
\frac{\lambda^{2} H}{1-\lambda H}\left\|u_{\mathrm{L}}\right\|_{2}<\left\|\mathrm{FH}\left(u_{\mathrm{L}}\right)\right\|_{2}
$$

satisfies Eq. (A·21), we can define the error bound for the HB method by

$$
\frac{\lambda^{2} H}{1-\lambda H}\left\|u_{\mathrm{L}}\right\|_{2}=\left\|\mathrm{FH}\left(u_{\mathrm{L}}\right)\right\|_{2}
$$

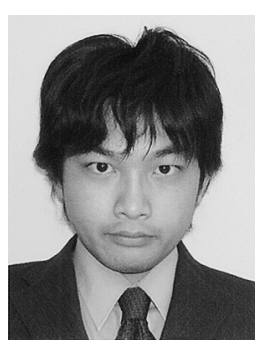

Masakazu Yagi received the B.E. and M.E. degrees in Electrical Engineering from Kyoto University, in 2003 and 2005, respectively. He is currently a Ph.D. student at Department of Electrical Engineering at Kyoto University. His research interests are nonlinear circuit.

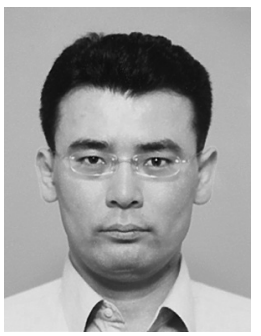

Takashi Hisakado received the B.E. and M.E. degrees in Electrical Engineering II from Kyoto University, in 1993 and 1995, respectively. He received the Dr. degree in Electrical Engineering from Kyoto University, in 1997. $\mathrm{He}$ is currently Associate Professor of Department of Electrical Engineering at Kyoto University. His research interests are nonlinear circuit, power circuit, EMC design, FPGA implementation, signal processing and so forth. He is a member of IEEJ, and IEEE.

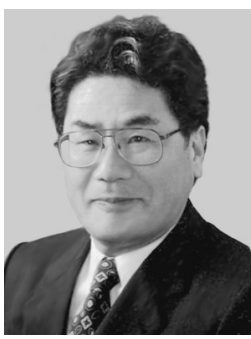

Kohshi Okumura was graduated from the Department of Electrical Engineering at Kyoto University, Kyoto, Japan, in 1966 and the master and doctor course of the graduate school at the same university in 1968 and 1971, respectively. From 1971 he worked as the instructor in the department of Electrical Engineering II at Kyoto University. Since 1992 he became a full professor at the same department. After reformation of the university in 1996, he was the professor at the graduate school of the same university. In 2004 he became the professor emeritus at Kyoto University. Currently he is a professor of the department of Information Science at Hiroshima Institute of Technology, Hiroshima, Japan. Supported by the Japan Ministry of Education he was appointed to a visiting researcher of London University (Queen Mary college) in 1989. He was the vice president of IEEJ as well as Engineering Science Society of IEICEJ. He was the chair of steering committee of NOLTA2005 and 2006 and also general co-Chair in NOLTA2007. His research interests are the analysis of nonlinear dynamical system and distributed transmission line. 